\title{
PTEN-regulated AKT/FoxO3a/Bim signaling contributes to reactive oxygen species-mediated apoptosis in selenite-treated colorectal cancer cells
}

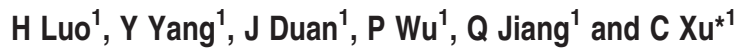

Mounting evidence shows that selenium possesses chemotherapeutic potential against tumor cells, including leukemia, prostate cancer and colorectal cancer (CRC) cells. However, the detailed mechanism by which sodium selenite specifically kills tumor cells remains unclear. Herein, we demonstrated that supranutritional doses of selenite-induced apoptosis in CRC cells through reactive oxygen species (ROS)-dependent modulation of the PI3K/AKT/FoxO3a signaling pathway. First, we found that selenite treatment in HCT116 and SW480 CRC cells caused inhibition of AKT and the nuclear accumulation of FoxO3a by western blot and immunofluorescence analyses, respectively, thereby facilitating transcription of the target genes bim and PTEN. Modulation of the AKT/FoxO3a/Bim signaling pathway by chemical inhibitors or RNA interference revealed that these events were critical for selenite-induced apoptosis in CRC cells. Additionally, we discovered that Fox03a-mediated upregulation of PTEN exerted a further inhibitory effect on the AKT survival pathway. We also corroborated our findings in vivo by performing immunohistochemistry experiments. In summary, our results show that selenite could induce ROS-dependent FoxO3a-mediated apoptosis in CRC cells and xenograft tumors through PTEN-mediated inhibition of the PI3K/AKT survival axis. These results help to elucidate the molecular mechanisms underlying selenite-induced cell death in tumor cells and provide a theoretical basis for translational applications of selenium.

Cell Death and Disease (2013) 4, e481; doi:10.1038/cddis.2013.3; published online 7 February 2013

Subject Category: Cancer

Selenium, an essential metalloid trace element, has been shown to possess chemopreventive and chemotherapeutic efficacy against multiple malignant cancers. ${ }^{1,2}$ For example, epidemiologic and preclinical data have shown an inverse relationship between selenium intake and cancer risk in humans. $^{3,4}$ However, the precise underlying molecular mechanisms responsible for these anticarcinogenic activities have not been resolved. Sodium selenite, a common form of inorganic selenium, was recently reported to induce apoptosis in several cancer cell lines. ${ }^{5-7}$ Our previous findings demonstrated that sodium selenite could specifically kill colorectal cancer (CRC) cells through the induction of apoptosis. ${ }^{8,9}$ In the present study, we further delineated the detailed mechanisms underlying seleniteinduced apoptosis.

Forkhead box $\mathrm{O}$ (FoxO) transcription factors are crucial regulators of diverse cellular activities, such as proliferation, differentiation, defense against oxidative stress, apoptosis and autophagy..$^{10,11}$ These factors are also associated with multiple diseases, including cancer. ${ }^{12,13}$ The FoxO family members include four highly related factors-FoxO1, FoxO3a, FoxO4 and FoxO6 ${ }^{14}$ - that can be posttranslationally regulated by various signaling molecules, of which AKT acts as an important upstream regulator. ${ }^{15}$ AKT directly phosphorylates FoxO family proteins and promotes their degradation. Consequently, less FoxO protein accumulates in the nucleus to execute protranscriptional actions towards target genes involved in cell-cycle arrest and apoptosis, such as bim, puma and p27. ${ }^{16-18}$ PI3K/AKT signaling has been shown to be frequently deregulated in various cancers, particularly in CRC. ${ }^{19,20}$ Therefore, exploration of the effects of sodium selenite on this signaling pathway and its involvement in apoptosis is of great significance for future clinical applications of selenium.

In the current study, we discovered that selenite conferred its proapoptotic effect through modulation of the PI3K/AKT/ FOXO3a signaling hub in both CRC cells and a colon xenograft model. We present clear evidence that sodium selenite inhibited the PI3K/AKT survival pathway in a reactive oxygen species (ROS)-dependent pathway. Furthermore, inhibition of AKT led to the activation of FoxO transcription factors and enhanced the expression of the target genes bim and PTEN; as a result, Bim was shown to promote seleniteinduced apoptosis, and PTEN amplified the proapoptotic effect of sodium selenite by inhibiting the AKT/FoxO3a/Bim signaling axis.

\footnotetext{
${ }^{1}$ National Laboratory of Medical Molecular Biology, Department of Biochemistry and Molecular Biology, Institute of Basic Medical Sciences, Peking Union Medical College and Chinese Academy of Medical Sciences, Beijing 100005, China

*Corresponding author: C Xu, Department of Biochemistry and Molecular Biology, Institute of Basic Medical Sciences, PUMC\&CAMS, Dongdan Santiao 5, Dongcheng District, Beijing 100005, China. Tel: + 8610 69156445; Fax: + 8610 69156445; E-mail: caiminxu@yahoo.com.cn

Keywords: selenite; apoptosis; AKT; FoxO3a; reactive oxygen species (ROS)

Abbreviations: FACS, fluoresence activated cell sorting; PTEN, phosphatase and tensin homolog; Bim, Bcl-2 interacting mediator of cell death

Received 10.09.12; revised 19.12.12; accepted 21.12.12; Edited by A Stephanou
} 


\section{Results}

Selenite-induced apoptosis is associated with the Src/PI3K/AKT/FoxO3a signaling axis. Following our previous study showing that supranutritional doses of selenite induced apoptosis in CRC cells, we aimed to elucidate the underlying molecular mechanisms. Thus, we conducted experiments to investigate whether selenite could influence the AKT survival pathway in CRC cells. As shown in Figure $1 \mathrm{a}$, we found that supranutritional doses of selenite time-dependently inhibited the SrC/PI3K/PDK1/AKT survival pathway in both HCT116 and SW480 CRC cells. Additionally, in vitro PI3K and AKT assays (Figure 1b; Supplementary Figure S1) showed that selenite treatment inhibited AKT and PI3K activation in HCT116 and SW480 CRC cells. We therefore postulated that FoxO family proteins may be regulated by selenite-inhibited AKT. To test this hypothesis, we immunoblotted FoxO family proteins in selenite-treated samples and found that selenite consistently suppressed the phosphorylation of these proteins (Figure 1c), indicating that FoxO proteins may be activated when AKT is inhibited by selenite. To further corroborate this finding, we extracted cytoplasmic and nuclear fractions from cells and immunoblotted for FoxO3a and p-Foxo3a in both control and selenite-treated samples and discovered that selenite increased the nuclear levels of FoxO3a but decreased its levels of phosphorylation (Figure 1d). Furthermore, immunofluorescence results (Figure 1e) also supported the above conclusion that selenite induced FoxO3a accumulation in the nucleus. Taken together, these results indicated that selenite inhibited SrC/PI3K/PDK1/AKT signaling and activated FoxO family proteins in HCT116 and SW480 CRC cells.

\footnotetext{
AKT/FoxO3a signaling is correlated with seleniteinduced apoptosis in CRC cells. Having discovered that selenite treatment inhibited Src/PI3K/PDK1/AKT signaling and activated FoxO proteins, we conducted a series of experiments to investigate the relationship between AKT and FoxO3a in selenite-induced apoptosis in CRC cells. On one hand, as revealed in Figures $2 a$ and $b$, when AKT was inhibited in selenite-treated CRC cells with either the PI3K inhibitor LY294002 or AKT siRNA, we found that both treatments further decreased the p-AKT level. As expected, inhibiting AKT further suppressed the phosphorylation of FoxO3a at Ser253 even with selenite treatment. Conversely, when we activated AKT in CRC cells using constitutively activated AKT constructs prior to selenite treatment, we found that, consistent with our hypothesis, constitutively activated AKT increased phosphorylation of AKT and FoxO3a and selenite could no longer reduce phosphorylation of AKT and consequently phosphorylation of FoxO3a (Figure 2c). These results collectively showed that seleniteelicited inhibition of AKT was associated with the activation of FoxO3a. Subsequently, we attempted to determine the role of AKT/FoxO3a in selenite-induced apoptosis of CRC cells. First, from western blot results of the above-mentioned samples, we observed that reactivation of AKT resulted in less cleavage of apoptosis-related markers such as caspase 9 and PARP, whereas further inhibition of AKT led to additional cleavage of these apoptosis-related markers.
}

Analysis of the apoptotic rate by FACS using cells treated as indicated in the panels of Figures $2 d$ and $e$ and Supplementary Figures S2A and B demonstrated that AKT reactivation or inhibition could blunt or enhance, respectively, the apoptosis of CRC cells treated with selenite. Complementary to the above results, silencing FoxO3a with siRNA specifically decreased the level of apoptosis in selenitetreated CRC cells, as revealed by western blotting and FACS (Figures $2 f$ and g; Supplementary Figure S2C). Thus, these findings clearly demonstrate that selenite induced apoptosis in CRC cells through regulation of the AKT/FoxO3a pathway.

Bim acts as a pivotal downstream factor of $\mathrm{FoxO}_{3} \mathrm{a}$ and thereby contributes to apoptosis. Accumulated FoxO3a in the nucleus can bind to promoters containing a consensus sequence to enhance the transcription of various molecules involved in apoptosis and the cell cycle, such as bim, puma, p27 and p21. ${ }^{21}$ Our previous results showed that Bcl-2 family proteins are critical regulators of selenite-induced apoptosis. ${ }^{22}$ Thus, we performed chromatin immunoprecipitation (ChIP) experiments to examine whether selenite could influence the binding of FoxO3a to the bim promoter to drive bim transcription. Indeed, as shown in Figure $3 a$, selenite treatment in HCT116 and SW480 CRC cells enhanced FoxO3a binding to the bim promoter, thus enhancing its transcription (Figure 3b). Accordingly, western blot results also showed that selenite treatment enhanced the expression of bim (Figure 3c). To explore whether Bim participated in selenite-induced apoptosis in CRC cells, we separated mitochondrial and cytoplasmic fractions from selenite-treated cells, immunoblotted for Bim and found that selenite treatment could induce the translocation of Bim from the cytoplasm to the mitochondria (Figure 3d). Moreover, immunostaining for Bim in HCT116 and SW480 CRC cells also corroborated the finding that selenite induced the colocalization of Bim with the mitochondria (Figure 3e). Finally, to further confirm the role of Bim in apoptosis, we knocked down the expression of Bim with siRNA in cells treated with selenite and found that Bim silencing markedly blocked selenite-induced apoptosis in HCT116 and SW480 CRC cells, as demonstrated by western blotting and FACS. (Figures $3 f$ and g; Supplementary Figure S3).

FoxO3a-upregulated PTEN expression is involved in regulating selenite-induced changes in the AKT/FoxO3a/ Bim signaling pathway. In our experiments, we unexpectedly found that selenite-induced FoxO3a also binds to the promoter of the PTEN gene (Figure 4a) in HCT116 and SW480 CRC cells, a finding also mentioned by Chiacchiera et al. ${ }^{23}$ Further experiments indicated that $\mathrm{FoxO} 3$ a directly facilitated PTEN transcription rather than blocking its degradation, as an mRNA synthesis inhibitor clearly inhibited the increase in PTEN mRNA after selenite treatment (Figure 4b). Moreover, the expression of PTEN also increased in a time-dependent manner after selenite treatment (Figure 4c). PTEN activity in selenite-treated cells was also enhanced in both cell lines (Figure 4d). To clarify whether upregulation of PTEN could indeed affect the AKT/ FoxO3a signaling pathway, we knocked down PTEN expression or transfected cells with a phosphatase-dead 
a
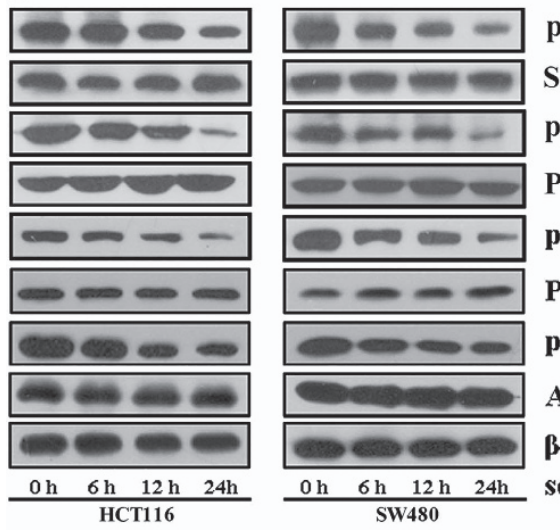

p-Src (Tyr 416)

Src

p-P85 (Tyr 458)

PI3K

p-PDK1 (Ser 241)

PDK1

p-AKT (Thr 308)

AKT

p-actin

selenite $(10 \mu \mathrm{mol} / \mathrm{L})$

c
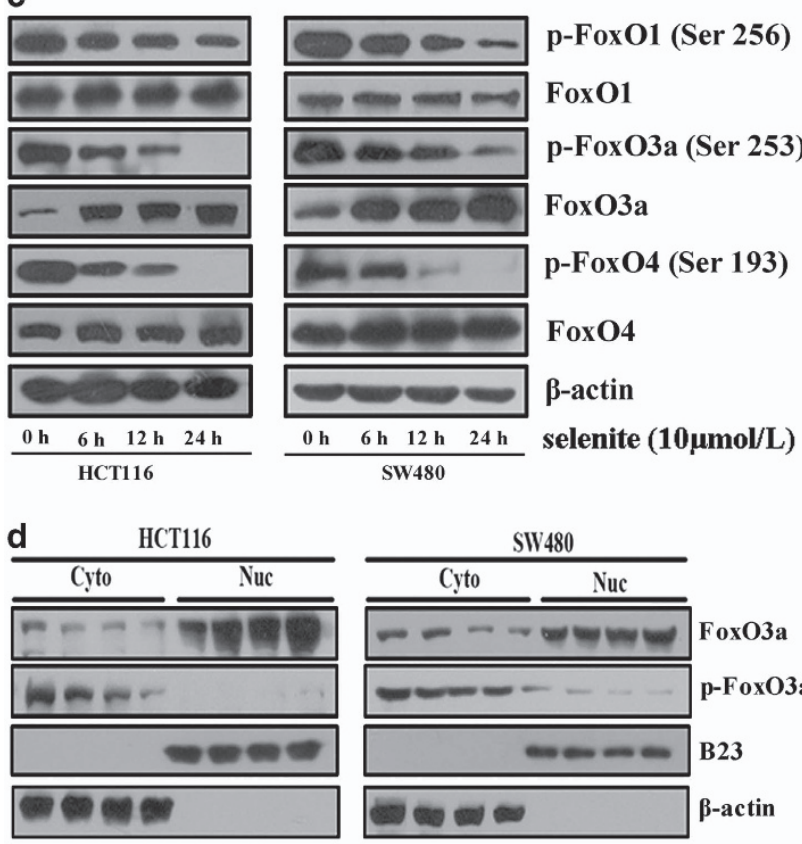

$0 \mathrm{~h} \quad 6 \mathrm{~h} \quad 12 \mathrm{~h} 24 \mathrm{~h} \quad 0 \mathrm{~h} \quad 6 \mathrm{~h} \quad 12 \mathrm{~h} 24 \mathrm{~h}$

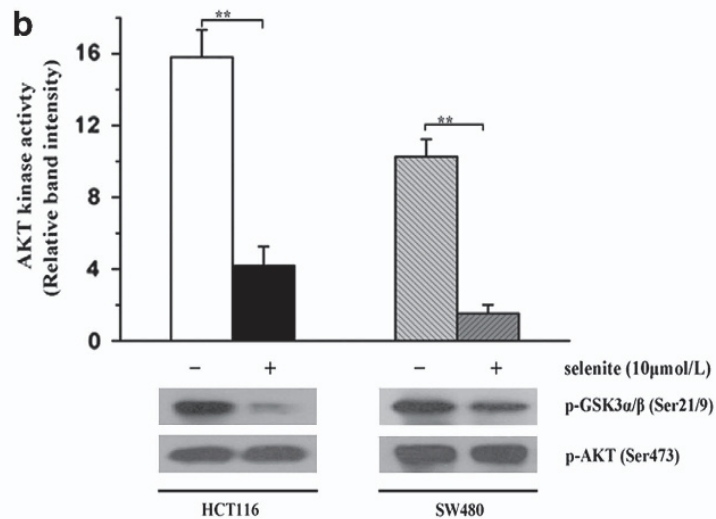

e
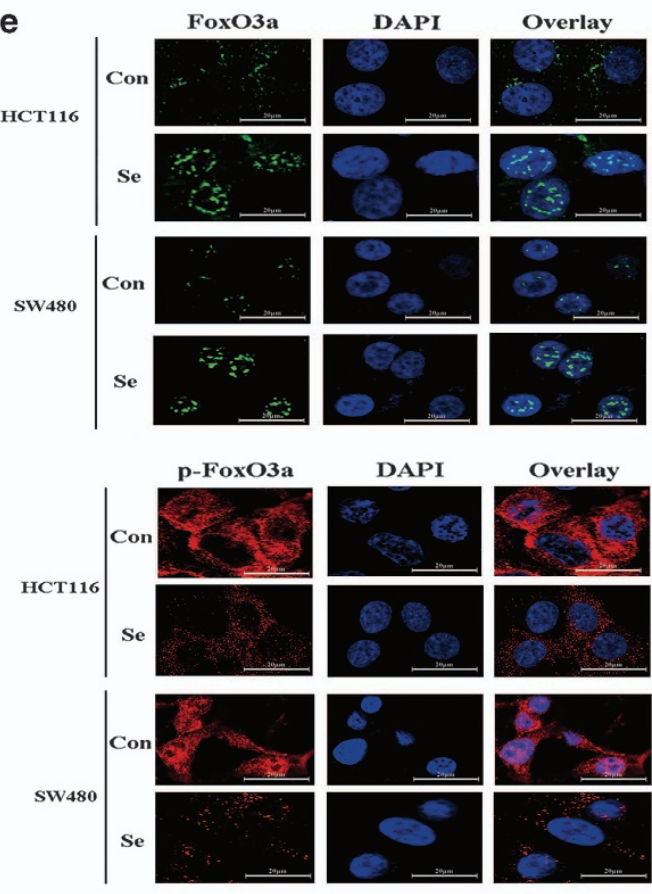

Overlay

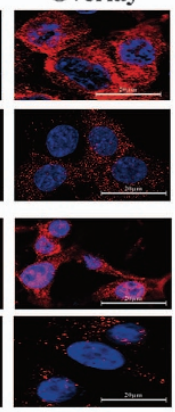

Figure 1 Selenite treatment caused time-dependent inhibition of PI3K/AKT and activation of FoxO proteins. (a) Selenite inhibited the Src/PI3K/PDK1/AKT signaling pathway. Cells were treated with $10 \mu \mathrm{mol} / \mathrm{l}$ selenite for various time periods as indicated and then immunoblotted for p-Src (Tyr 416), Src, p-p85 (Tyr458), p85, p-PDK1 (Ser241), PDK1, p-AKT (Thr308) and AKT. $\beta$-Actin was probed to ensure equal protein loading. (b) AKT kinase activity was inhibited by selenite. HCT116 and SW480 cells were treated with either sodium selenite or PBS solution for $24 \mathrm{~h}$. Subsequently, cells were collected after trypsinization and total cellular cell lysates were prepared using RIPA buffer. p-AKT was captured using from the lysates and the in vitro kinase reaction was performed as described in the Materials and Methods section. The relative intensity of the p-GSK3 $\alpha / \beta$ band reflected the AKT kinase activity and p-AKT was immunoblotted as controls. AKT activity was calculated among groups. The error bars represent the mean values \pm S.D. from at least three independent experiments $\left({ }^{* *} P<0.01\right)$. (c) Selenite decreased the phosphorylation of FoxO family proteins and increased the expression of total FoxO proteins. HCT116 and SW480 CRC cells were treated with either selenite or PBS solution for the indicated time periods. Total cellular lysates were subjected to western blot analysis using antibodies against p-FoxO1(Ser256), FoxO1, p-FoxO3a (Ser253), FoxO3a, p-FoxO4 (Ser193) and FoxO4. $\beta$-Actin was used as a loading control. (d) Selenite treatment induced dephosphorylation of Fox03a and its nuclear accumulation. Nuclear and cytoplasmic proteins were fractioned from selenitetreated HCT116 and SW480 CRC cells and were then subjected to western blot analysis using antibodies against FoxO3a and p-FoxO3a (Ser253). B23 and $\beta$-actin were blotted to demonstrate the purity of each fraction. (e) Foxo3a and p-Foxo3a proteins in selenite-treated or control cells were immunostained with primary antibodies and the corresponding FITC- or Cy3-conjugated secondary antibodies followed by detection using confocal microscopy. Green signals indicate Foxo3a, whereas red signals indicate p-Fox03a. Nuclei were counterstained with DAPI. Representative images of each sample are shown

(C124S) mutant. As shown in Figures $4 \mathrm{e}$ and $\mathrm{f}, \mathrm{PTEN}$ knockdown reversed the changes elicited by selenite in both cell lines. In addition, the inhibition of PTEN by SF $1670^{24}$ abrogated the changes in the AKT/FoxO3a/Bim pathway induced by upregulated PTEN (Figure 4g). From these results, we concluded that selenite-induced inhibition of AKT and the activation of $\mathrm{FoxO} 3 \mathrm{a} / \mathrm{Bim}$ as well as apoptosis were critically regulated by increased levels of PTEN.

Selenite-induced ROS are indispensable for AKT/ FOXO3a/Bim-mediated apoptosis in CRC cells. Previous work, including our own, has identified ROS as an important 
a

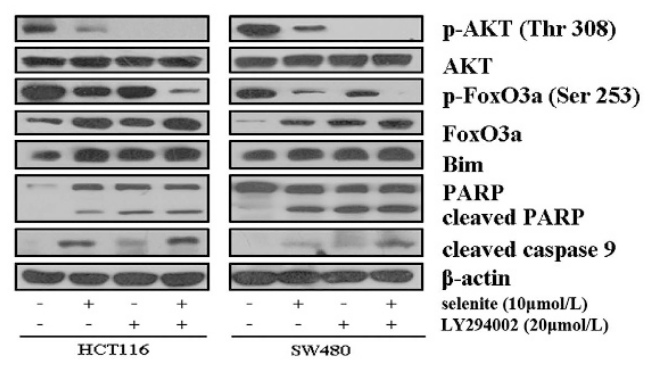

b

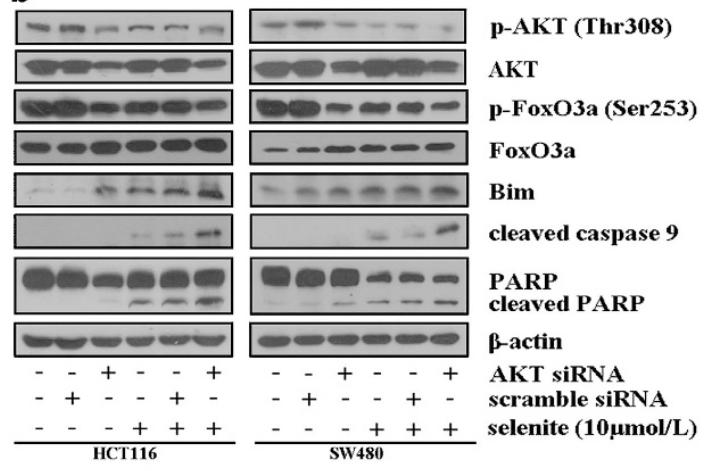

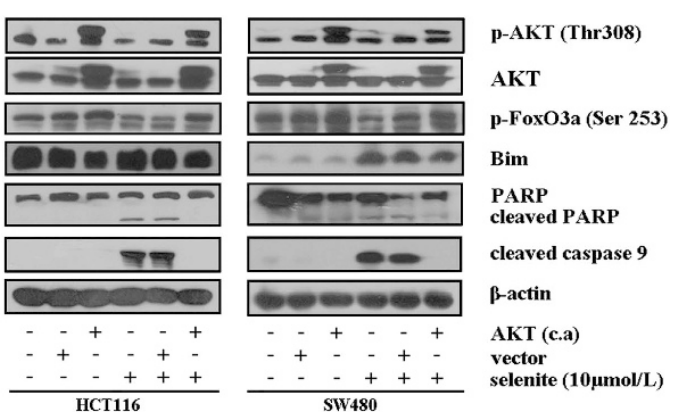

f

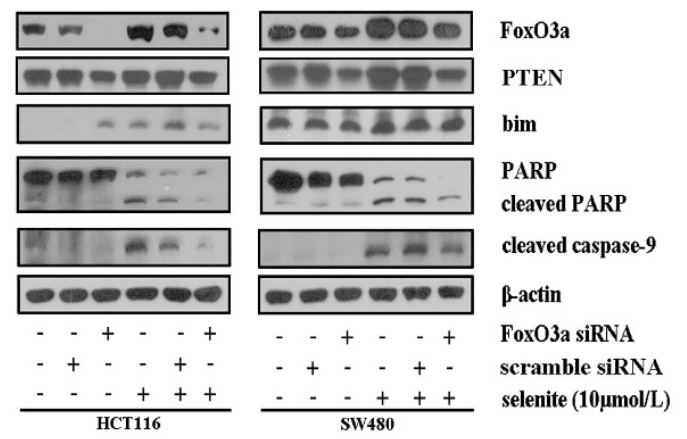

d
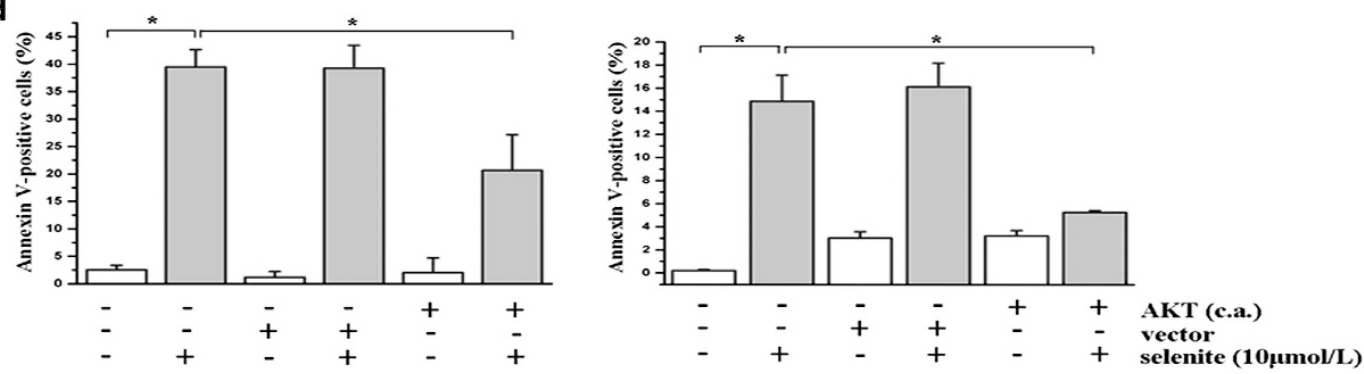

e
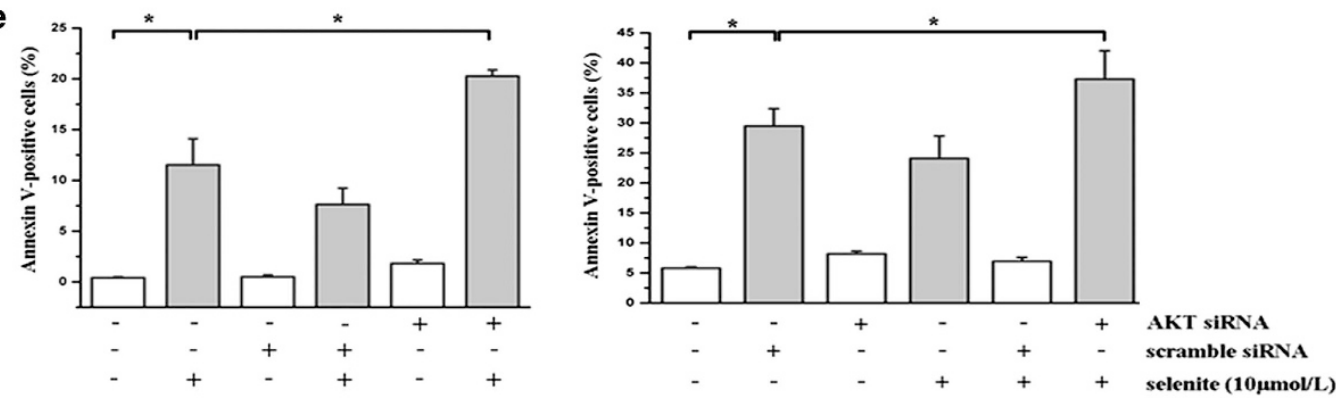

g
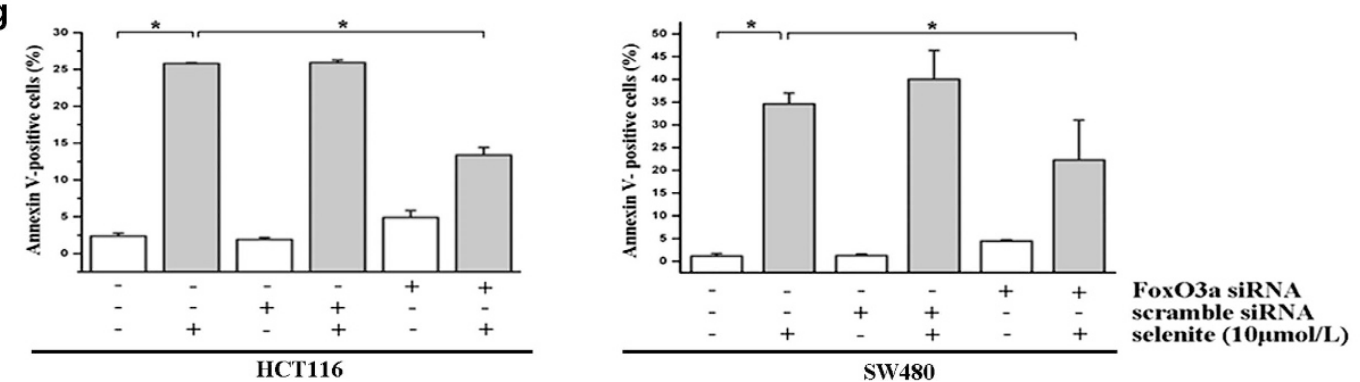
factor in the induction of apoptosis in cancer cells. ${ }^{25-27}$ Our previous work showed that sodium selenite treatment could induce an increased level of ROS in CRC cells. ${ }^{9}$ Thus, we conducted experiments to elucidate whether ROS were involved in selenite-induced apoptosis in CRC cells. To explore the possible link between ROS and AKT/FOXO3a/ Bim-mediated apoptosis, we eliminated ROS in selenitetreated cells using a MnSOD mimic, the widely used ROS scavenger MnTMPyP or another ROS extinguisher (Tiron) and found that depletion of ROS almost completely blocked apoptosis induced by selenite, as observed by the disappearance of cleaved PARP. Furthermore, this signaling pathway regulated by selenite that was also relieved by ROS depletion strongly argues for a role of ROS in seleniteinduced AKT/FOXO3a/Bim-mediated apoptosis in CRC cells (Figure 5a).

The PTEN/AKT/FoxO3a/Bim signaling pathway is regulated by selenite in vivo. Having defined the role of PTEN/ AKT/FoxO3a/Bim signaling in selenite-induced apoptosis in $\mathrm{CRC}$ cells, we sought to test whether selenite could regulate this signaling pathway in vivo. We previously observed that selenite treatment could markedly inhibit tumor growth and induce apoptosis in a SW480 colon xenograft model. ${ }^{8}$ To verify these results in additional tissues, we first performed western blot analysis of tissues from both control and selenite-treated samples, and the results revealed that selenite could inhibit the phosphorylation of PI3K/PDK1/AKT and FoxO3a, thereby upregulating $\mathrm{Bim}$ and PTEN (Figure 5b). Additionally, in a series of immunohistochemistry experiments, we examined the expression patterns of critical molecules in this signaling pathway, including p-AKT, AKT, FoxO3a, p-FoxO3a, Bim and PTEN, and discovered that each of these proteins displayed a similar pattern as that seen in tumor cell lines (Figure 5c; Supplementary Figure S5).

\section{Discussion}

The present study presents evidence that the AKT/FoxO3a/ Bim/PTEN signaling axis is closely associated with seleniteinduced apoptosis in CRC cells and xenograft tumors. A model depicting our findings is shown in Figure 6. Together, our results suggest that supranutritional doses of selenite inhibit Src/PI3K/PDK1/AKT signaling and activate FoxO proteins. Further experiments revealed that inhibiting or activating AKT genetically or pharmacologically together with selenite treatment resulted in the further regulation of FoxO3a as well as its target bim. We also confirmed that seleniteinduced activation of FoxO3a could enhance the transcription of bim and PTEN via increased promoter binding of FoxO3a. Enhanced levels of bim were further shown to translocate from the cytoplasm to mitochondria, which played a crucial role in the activation of caspase 9 and PARP resulting from selenite treatment. Furthermore, we found that FoxO3ainduced PTEN played a role in the selenite-regulated AKT/ FoxO3a/Bim signaling pathway, further amplifying the proapoptotic effect of selenite. Moreover, depletion of ROS via treatment with MnTmPyP or Tiron in selenite-induced cells reversed the changes observed in the $\mathrm{AKT} / \mathrm{FoxO} 3 \mathrm{a} / \mathrm{Bim}$ signaling pathway, implying that ROS may be involved in selenite-induced regulation of the AKT/FoxO3a/Bim signaling pathway in HCT116 and SW480 CRC cells.

FoxO family proteins have emerged as master regulators that control a plethora of cellular activities through the orchestration of different patterns of gene expression in response to diverse stimuli. ${ }^{28}$ Notably, studies by the group of David $\mathrm{T}$ Scadden revealed a role for FoxO3a in maintaining a differentiation blockade in AML cells, ${ }^{29}$ which is in contrast to its canonical tumor suppressor role. Furthermore, these cells could be regulated by many upstream factors such as AKT, ERK, IKK $\beta$ and JNK under different contexts. ${ }^{30-33}$ In the present study, we focused on the effect of AKT on FoxO3a and its downstream targets because AKT was shown to be aberrantly expressed in multiple malignant tumors, particularly in CRC. Thus, exploring the molecular mechanisms of drugs targeting AKT could be of great significance for treating cancer, particularly for tumors harboring aberrantly upregulated AKT activity. First, we found that selenite inhibited AKT and its canonical upstream regulator PI3K and PDK1. We demonstrated that AKT inhibition directly activated FoxO3a in response to selenite, an event crucial for selenite-induced apoptosis. The AKT/FoxO3a signaling hub has also been shown to be regulated by many other chemotherapy drugs, such as 18 $\beta$-glycyrrhetinic acid, isoflavone and paclitaxel. ${ }^{34-36}$ FoxO3a is phosphorylated by AKT at Thr32, Ser256 and Ser319, and phosphorylation of these amino acids provides binding sites for 14-3-3 proteins, resulting in the retention of FoxO3a by 14-3-3 in the cytoplasm. Accordingly, selenite treatment drastically decreased 14-3-3 binding sites on FoxO3a proteins, indicating that $\mathrm{FoxO} 3 \mathrm{a}$ was retained in the nucleus (Supplementary Figure S2D). Furthermore, inhibition of AKT by selenite was shown to be directly related to the reduced phosphorylation of FoxO3a (Figures $2 a$ and b), which resulted in FoxO3a accumulation in the nucleus. This prompted us to further investigate the role of FoxO3a in the nucleus following treatment with selenite in CRC cells.

Bim is widely known for its pro-apoptotic functions in mitochondria, and it induces apoptosis by interacting with

Figure 2 Selenite-induced inhibition of AKT directly activated FoxO3a in CRC cells, an event closely correlated with apoptosis. (a, b) Inhibition of AKT with either LY294002 or AKT siRNA led to an inhibition of FoxO3a and increased apoptosis in selenite-treated HCT116 and SW480 CRC cells. Cells were treated with LY294002 for $1 \mathrm{~h}$ prior to selenite treatment or were transfected with AKT siRNA followed by treatment with either selenite or PBS for $24 \mathrm{~h}$. Cells were then collected, and total cellular lysates were immunoblotted for p-AKT (Thr308) and p-Foxo3a (Ser253), Bim, cleaved PARP and cleaved caspase 9 and $\beta$-actin. (c) Activation of AKT protected cells from seleniteinduced apoptosis and modulation of AKT/FoxO3a/Bim signaling. HCT116 and SW480 CRC cells were transfected with constitutively activated (c.a.) AKT constructs to restore AKT prior to selenite treatment for $24 \mathrm{~h}$ and were then subjected to western blot assays using antibodies against p-AKT, AKT, p-FoxO3a, Bim, cleaved PARP and cleaved caspase 9. $\beta$-Actin was used as a loading control. (d, e) The apoptotic rate of cells was determined by FACS in cells transfected with constitutively activated (c.a.) AKT constructs or AKT siRNA and treated with selenite. The results were calculated and are statistically represented as bar graphs. ${ }^{*} P<0.05$ indicates statistical significance between groups. $(\mathbf{f}, \mathbf{g})$ Foxo3a-mediated selenite-induced apoptosis downstream of AKT. Cells were transfected with FoxO3a siRNA prior to treatment with selenite for $24 \mathrm{~h}$, after which time apoptosis was analyzed using western blotting or FACS 
a

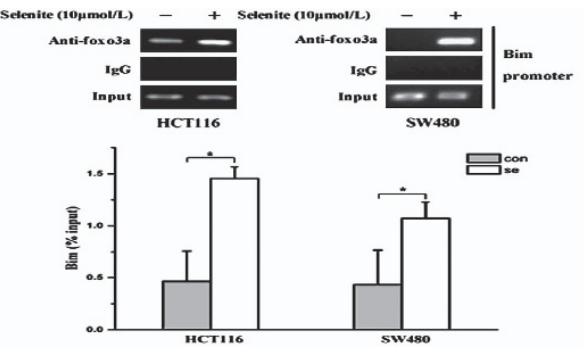

b

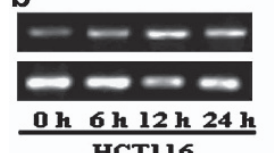
HCT1 16

C

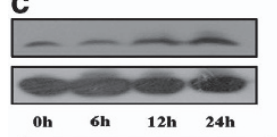

Oh $\quad 6$ h $\quad 12 h \quad 24 h$

HCT116

f
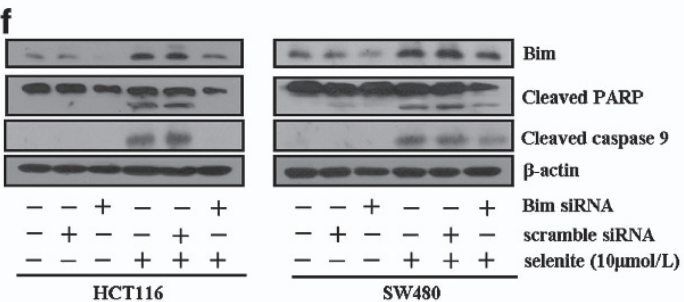

g

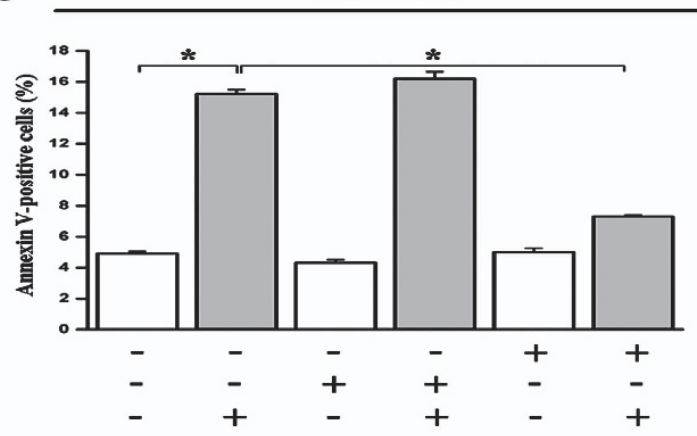

d

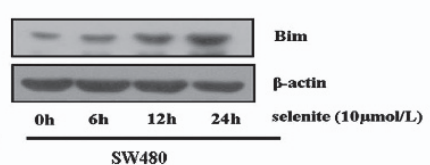

HCT116
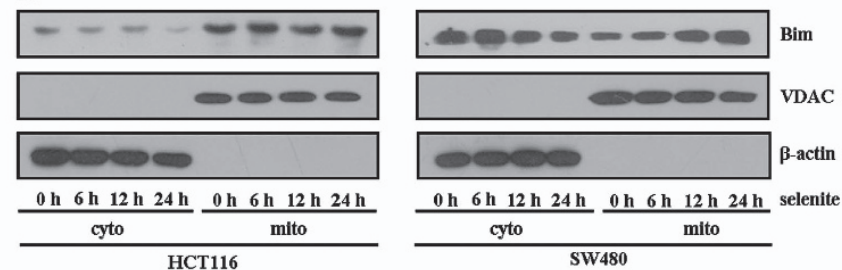

0h 6 h 12 h 24 h 0 h 6 h 12 h 24 h selenite $(10 \mu \mathrm{mol} / \mathrm{L})$ cyto SW480

e

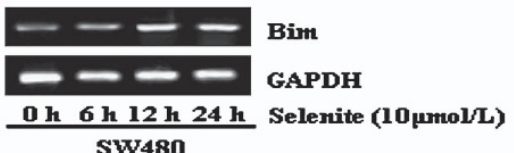

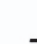

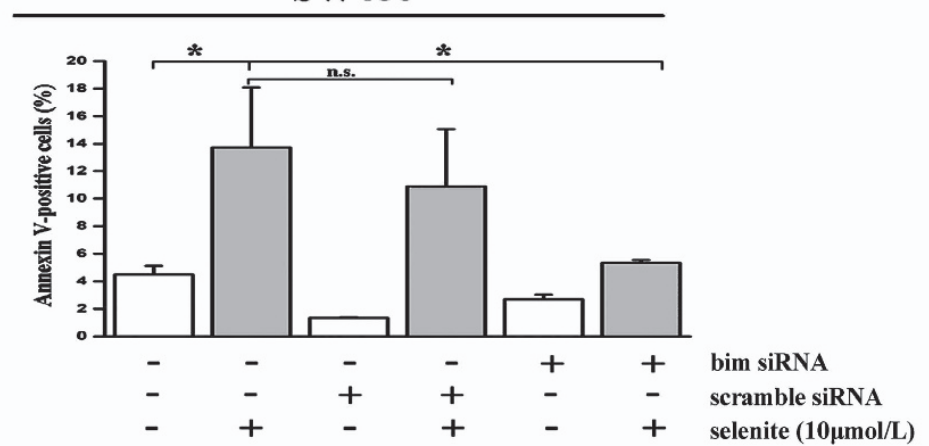

Figure 3 FoxO3a promoted the transcription and expression of bim, thereby enhancing apoptosis. (a) Selenite facilitated FoxO3a binding to the bim promoter. HCT116 and SW480 CRC cells were treated with or without selenite for $24 \mathrm{~h}$ followed by subjection to the ChIP assay. The relative band intensity of PCR products revealed the binding of FoxO3a to the bim promoter. (b, c) Selenite increased the transcription of bim in both HCT116 and SW480 CRC cells. Cells were treated with selenite for the indicated time periods followed by reverse transcription PCR and western blotting. (d) Selenite treatment caused the translocation of Bim from the cytoplasm to the mitochondria. HCT116 and SW480 CRC cells were treated with selenite for the indicated time periods, and the mitochondria were then isolated and immunoblotted for Bim. VDAC and $\beta$-actin were used as markers of the mitochondria and cytoplasm, respectively. (e) Colocalization results of Bim in selenite-treated HCT116 and SW480 CRC cells. Cells were treated with selenite for $24 \mathrm{~h}$, and Mitotracker (red) solution was added to the medium to stain the mitochondria in cells. Cells were collected and stained with a Bim primary antibody and a FITC-conjugated secondary antibody (green). Nuclei are shown as blue signals. (f, g) Knockdown of Bim attenuated apoptosis in selenite-treated CRC cells. Bim siRNA was used to silence the expression of bim in HCT116 and SW480 CRC cells that subsequently were treated with selenite for $24 \mathrm{~h}$. All groups of cells were analyzed using western blotting and FACS

proteins harboring anti-apoptotic function such as $\mathrm{Bcl}-\mathrm{xL}$ and $\mathrm{Bcl}-2$. Such interactions release proteins, including Bax and Bak, at the mitochondria to initiate apoptosis. ${ }^{37,38}$ Bim was also shown to be a direct target of $\mathrm{FoxO} 3 \mathrm{a}^{39}$ In the present study, we found that activated FoxO3a could bind more intensely to the promoter of bim, thereby facilitating bim transcription. In parallel, an increased Bim level was correlated with translocation from the cytoplasm to the mitochondria, and knockdown experiments showed that selenite-induced bim expression was involved in seleniteinduced apoptosis.

PTEN is commonly mutated in various cancers, ${ }^{40,41}$ as it normally functions as a tumor suppressor to antagonize the effects of PI3K through its lipid phosphatase activity. 
a

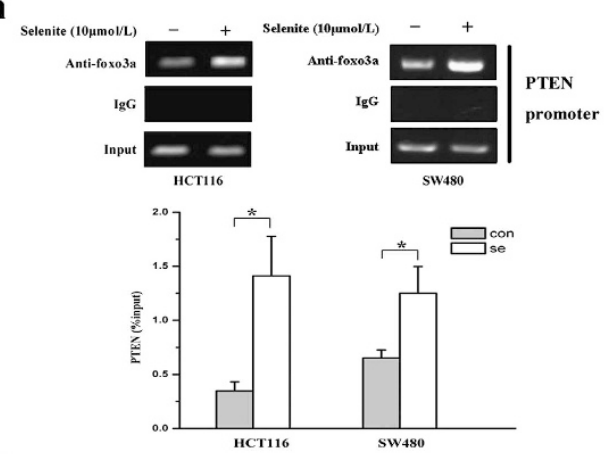

C
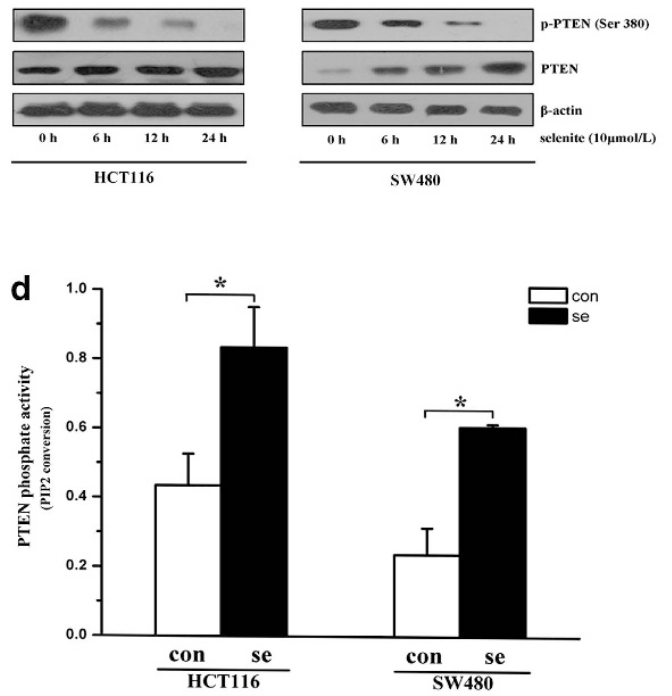

f

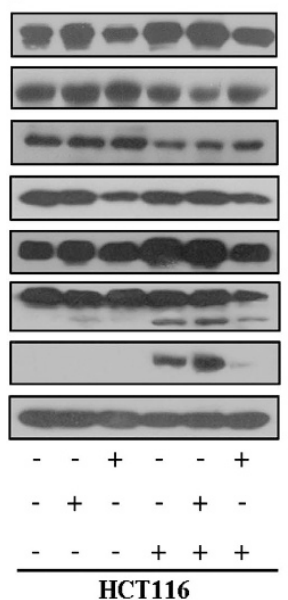

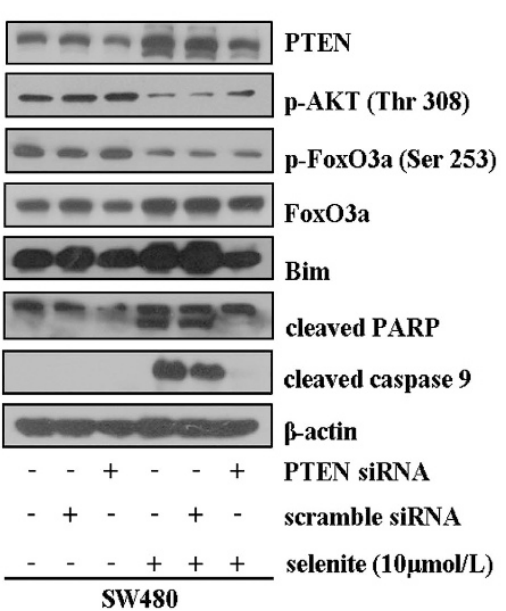

b
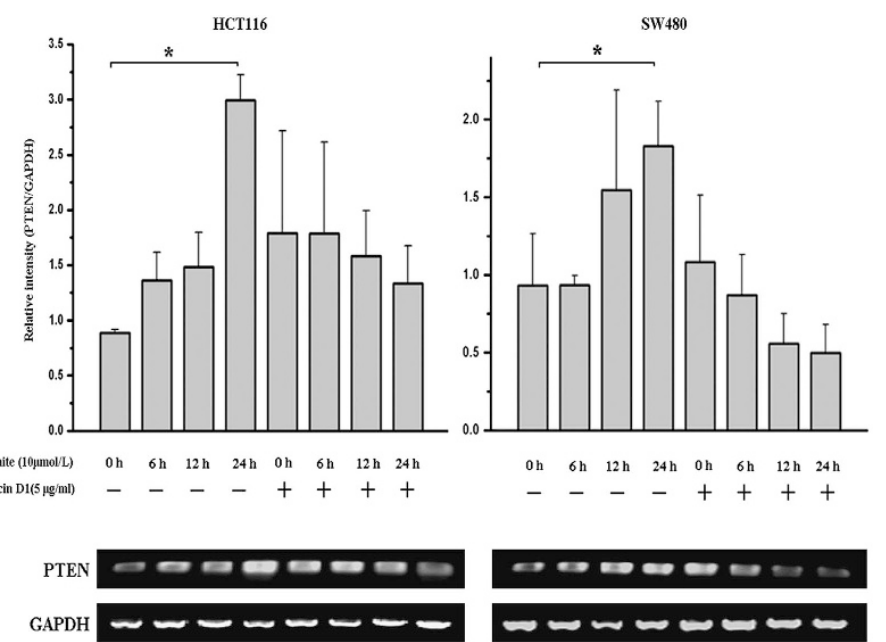

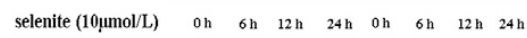
actinomycin D1 $(5 \mu \mathrm{g} / \mathrm{ml}) \quad-\quad-\quad-\quad+\quad++$

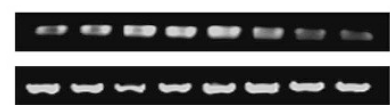

$\begin{array}{lllllllllllll}0 \mathrm{~h} & 6 \mathrm{~h} & 12 \mathrm{~h} & 24 \mathrm{~h} & 0 \mathrm{~h} & 6 \mathrm{~h} & 12 \mathrm{~h} & 24 \mathrm{~h}\end{array}$ e

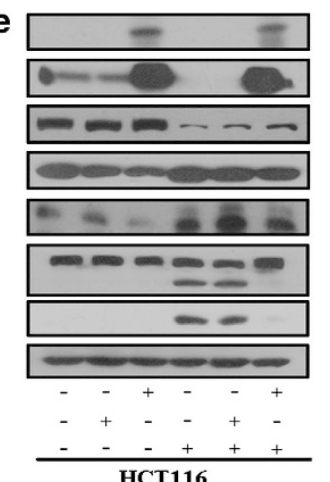

g

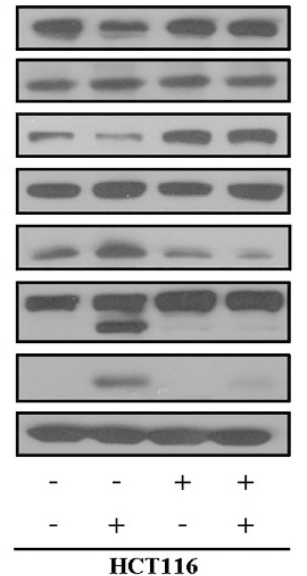

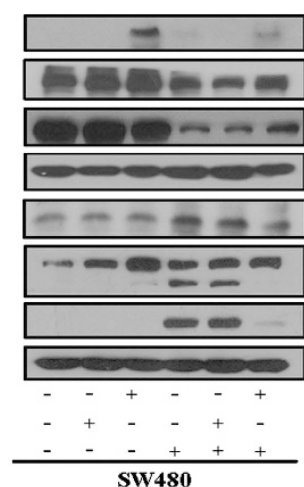

HA-PTEN(C124S)

p-AKT(Thr-308)

p-FoxO3a (Ser 253)

FoxO3a

Bim

PARP

cleaved PARP

cleaved caspase 9

B-actin

PTEN c124s

vector selenite $(10 \mu \mathrm{mol} / \mathrm{L})$

SW480

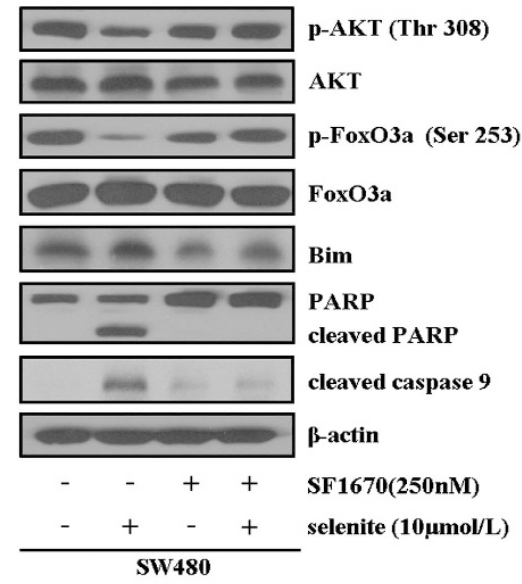

Figure 4 Selenite-induced PTEN modulated the AKT/FoxO3a/Bim signaling pathway. (a) Selenite treatment facilitated the binding of FoxO3a to the PTEN promoter. HCT116 and SW480 CRC cells were treated with selenite for $24 \mathrm{~h}$ and then were subjected to $\mathrm{ChIP}$ analysis. The binding ability of FoxO3a to the PTEN promoter was calculated and analyzed. (b) Selenite treatment enhanced the synthesis of PTEN mRNA through FoxO3a accumulation in the nucleus. HCT116 and SW480 CRC cells were treated for the indicated time periods with or without actinomycin D1 to inhibit new mRNA synthesis. Total cellular mRNA was then extracted and subjected to reverse transcription PCR. The PTEN mRNA level was determined and calculated from three independent experiments. (c) The expression of PTEN was enhanced in selenite-treated CRC cells. Western blotting was performed to determine the expression of PTEN in HCT116 and SW480 CRC cells after selenite treatment. (d) Selenite enhanced PTEN phosphatase activity in HCT116 and SW480 CRC cells. Twenty-four hours after selenite treatment, cells were collected, and PTEN phosphatase activity in each sample was determined as described in the Materials and Methods section. (e, f) PTEN perturbed the selenite-regulated AKT/FoxO3a signaling axis. Cells were transfected with phosphatase-dead PTEN (C124S) plasmids or PTEN siRNA to efficiently extinguish PTEN activity. Subsequently, cells were treated with or without selenite for $24 \mathrm{~h}$, and then the expression levels of p-AKT (Thr308), p-FoxO3a (Ser253), FoxO3a, Bim, cleaved PARP and cleaved caspase 9 were detected using western blotting. $\beta$-Actin was used as a loading control. $(\mathbf{g})$ Inhibition of PTEN abrogated the further inhibitory effect of PTEN on the AKT/FoxO3a/Bim signaling pathway. HCT116 and SW480 cells were treated with SF1670, a PTEN inhibitor, followed by selenite or PBS for $24 \mathrm{~h}$. The altered expression patterns of p-AKT, AKT, p-FoxO3a, FoxO3a, cleaved PARP and cleaved caspase 9 were determined using western blotting. $\beta$-Actin was used as a control for equal loading 

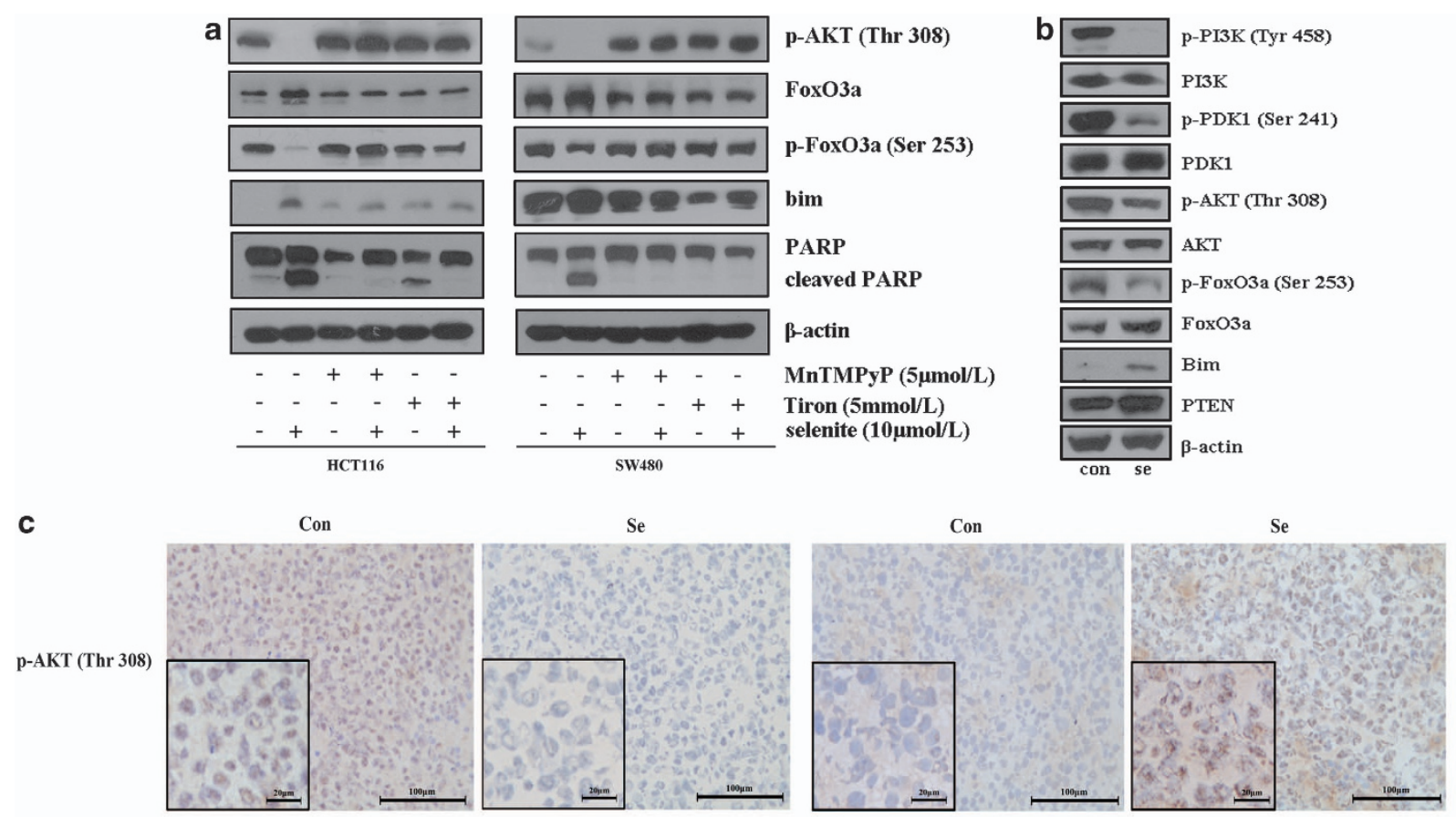

FOX03a
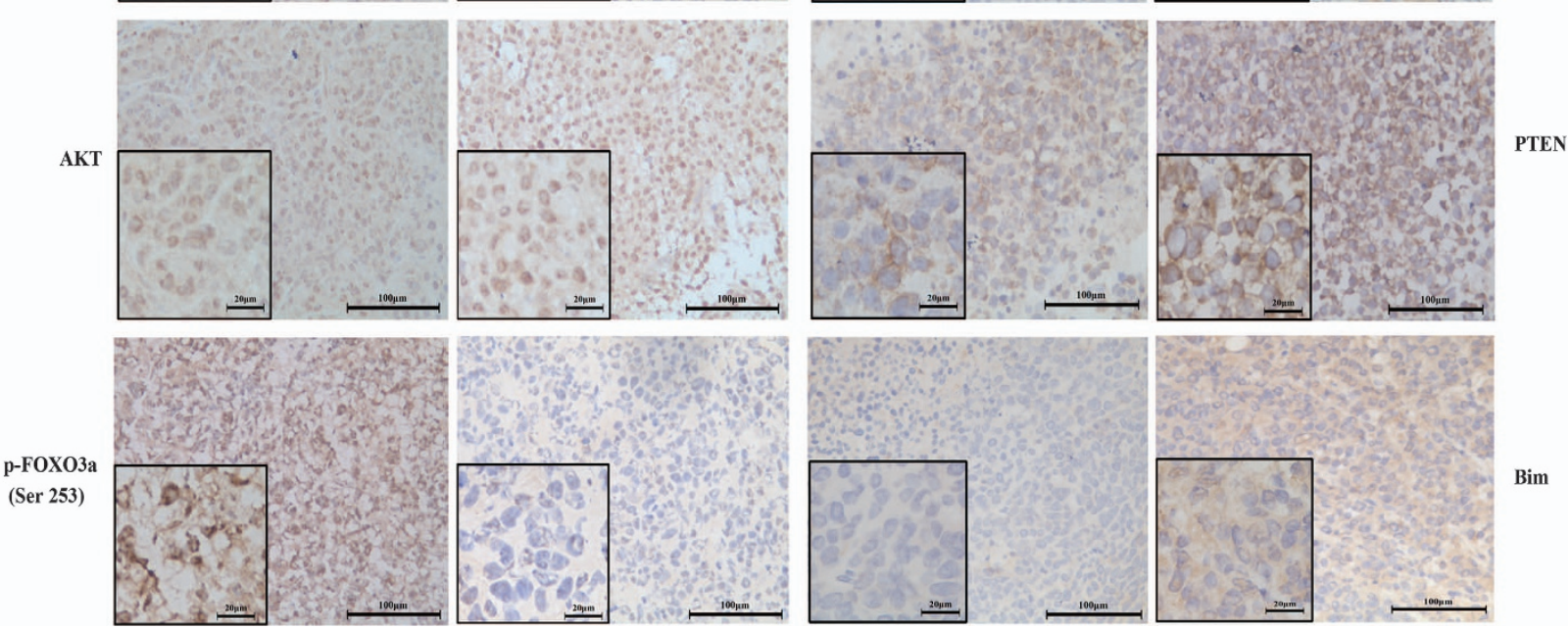

Figure 5 (a) ROS were involved in selenite-mediated regulation of the AKT/FoxO3a/Bim signaling pathway in HCT116 and SW480 CRC cells. Cells samples were pretreated with MnTmPyP or Tiron solution and then were treated with selenite or PBS for $24 \mathrm{~h}$. Cell lysates were analyzed by western blot for the molecules shown in the figures. (b) Western blot analysis of proteins extracted from tumor tissues using antibodies against critical molecules as indicated. (c) Selenite-mediated regulation of the PTEN/AKT/FoxO3a/Bim signaling pathway in vivo. Tumor tissues from a colon xenograft animal model were subjected to immunohistochemistry analysis using antibodies recognizing p-AKT (Thr308), AKT, p-FoxO3a (Ser253), FoxO3a, PTEN and Bim. Representative images from both control and selenite-treated samples are shown

Consequently, AKT activation is balanced by both PTEN and PI3K. In the present study, we observed that selenite inhibited the phosphorylation of Src and the p85 subunit of PI3K and its downstream effectors PDK1 and AKT. Additionally, PTEN expression was upregulated by FoxO3a and (Figures $2 f$ and $4 \mathrm{c}$ ), and PTEN activity was enhanced in response to selenite treatment (Figure 4d). These findings are supported by work from Meuillet and coworkers. ${ }^{42}$ Therefore, we hypothesized that selenite-induced activation of PTEN was involved in regulation of the AKT/FoxO3a/Bim signaling pathway. We transfected cells with lipid phosphatase-dead PTEN plasmids or PTEN siRNA as well as inhibiting PTEN with SF1670 and discovered that selenite-mediated modulation of the AKT/FoxO3a/Bim pathway was abrogated when
PTEN was inhibited. Furthermore, activating PTEN with NaBT in HCT116 and SW480 CRC cells exerted further inhibitory effects on the AKT/FoxO3a/Bim signaling pathway (Supplementary Figure S4). We concluded that seleniteinduced PTEN was associated with the AKT/FoxO3a/Bim pathway and apoptosis in HCT116 and SW480 CRC cells, which is consistent with the findings from other groups showing that PTEN directly regulates AKT/FoxO3a under various circumstances. ${ }^{43,44}$ However, whether a positive feedback loop exists between PTEN and the AKT/FoxO/Bim signaling pathway requires further study.

Our previous results, along with the findings of other studies, have implicated ROS as a potential mediator of selenite-induced apoptosis and its related signaling pathway 


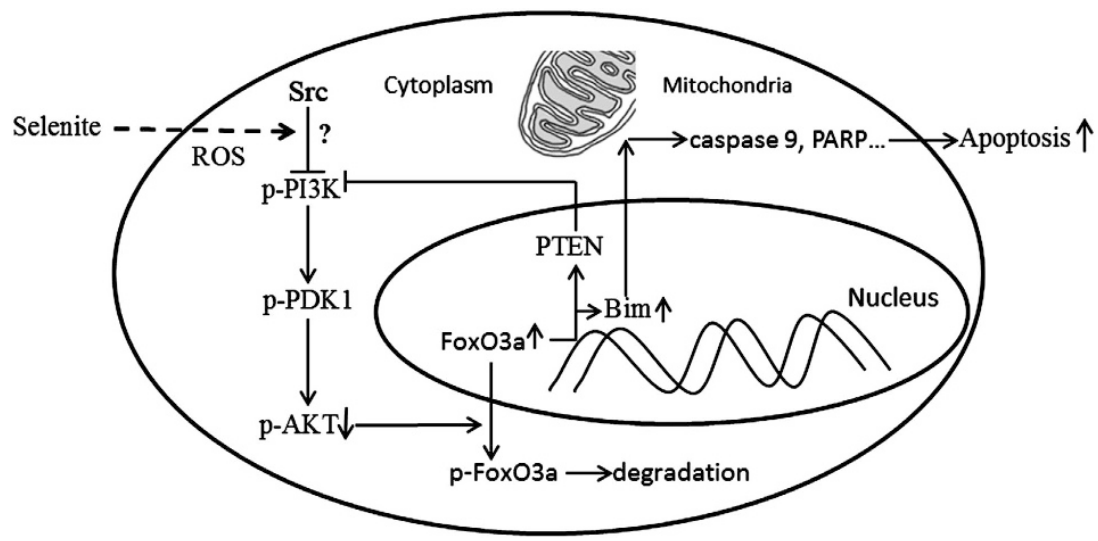

Figure 6 A schematic illustration delineating the role of the AKT/Foxo3a/Bim pathway in selenite-induced apoptosis of CRC cells. Selenite treatment inhibited Src/PI3K/ PDK1/AKT pathway and accumulation of FoxO3a in the nucleus possibly through induced ROS in CRC cells. On one hand, upregulated FoxO3a enhanced the transcription and expression of bim, which led to apoptosis. Additionally, PTEN was also upregulated by FoxO3a, indicating further regulation of AKT/FoxO3a/Bim signaling and apoptosis in response to selenite

in tumor cells. ${ }^{5,7,9}$ To define the role of selenite-induced ROS in the AKT/FoxO3a/Bim signaling pathway, we inhibited selenite-induced ROS in CRC cells and observed that the above change in the AKT/FoxO3a/Bim pathway was blocked completely. Additionally, selenite-induced apoptosis was blunted when cells were pretreated with ROS scavengers. Thus, the selenite-regulated PTEN/AKT/FoxO3a/Bim signaling hub and apoptosis are critically modulated by ROS in HCT116 and SW480 cells. However, much work still needs to be done to clarify the relationship between ROS and selenitemodulated FoxO proteins, as work by Schulze coworkers ${ }^{45}$ found that FoxO proteins could reduce the ROS level in cells by impairing the expression of genes with mitochondrial function rather than in the canonical SOD2-independent manner. Moreover, work by Yoon et al. ${ }^{46}$ concluded that selenite inhibits apoptosis through activation of PI3K/AKT signaling, and Xiangjia Zhu together with his colleagues discovered that selenite inhibits 1,2-dihydroxynaphthaleneinduced apoptosis in human lens epithelial cells through activation of the PI3K/Akt pathway. ${ }^{47}$ However, it should be noted that the concentration of selenite used in these previous studies was very low $(2-3 \mu \mathrm{mol} / \mathrm{l})$ and was a physiological dose that caused different effects regarding cell survival and the PI3K/Akt signaling pathway. Our previous study also provided evidence that low-dose selenite could promote cell survival, whereas supranutritional doses of selenite could induce apoptosis in CRC cells. ${ }^{48}$ The effects of selenite on cell fate and regulation of this signaling axis depend on the doses and different cellular systems, which also applies to in vivo experiments. Although we used a colon xenograft model to strengthen our findings, we paid much attention to the heterogeneity of cancer cells when reaching our conclusion. Thus, much work needs to be done to define the role of selenite in vivo, which is a main focus of our future research.

In summary, the ensemble of evidence presented in the current study demonstrates that sodium selenite could induce apoptosis specifically in CRC cells by inhibiting Src/PI3K/AKT survival factors and activating FoxO proteins along with the targets bim and PTEN. Activated FoxO3a bound more intensely to the Bim and PTEN promoters, thereby enhancing their transcription and expression, and our immunofluorescence and western blot results both demonstrated that increased levels of Bim translocated from the cytoplasm to the mitochondria. Furthermore, RNA interference experiments revealed that this process was essential for selenite-induced apoptosis. Seleniteinduced PTEN further amplified this effect on the AKT/FoxO3a/ Bim signaling pathway. However, whether selenite can directly affect PTEN activity through somehow mechanisms warrants further study. These findings help to elucidate the molecular effects of selenite treatment and provide a theoretical basis for its clinical application, and exploration of the detailed molecular mechanisms underlying the efficacy of selenite in treating malignant cancer is of great significance for translational medicine.

\section{Materials and Methods}

Cell lines and culture. The human CRC cell lines HCT116 and SW480 were purchased from the cell culture center of the Institute of Basic Medical Sciences, Chinese Academy of Medical Sciences and maintained in DMEM (Invitrogen, Paisley, Scotland) supplemented with $10 \%$ fetal bovine serum (FBS; Hyclone, Logan, UT, USA), $100 \mathrm{units} / \mathrm{ml}$ penicillin and $100 \mu \mathrm{g} / \mathrm{ml}$ streptomycin in a humidified $5 \% \mathrm{CO}_{2}$ atmosphere at $37^{\circ} \mathrm{C}$. All cell cultures were monitored routinely, all cell lines were discarded after 2 months, and new lines were propagated from the frozen stocks.

Reagents and antibodies. Sodium selenite, Tiron, NaBT and SF1670 were purchased from Sigma-Aldrich (St. Louis, MO, USA). A $10 \mathrm{mmol} / \mathrm{I}$ stock solution of selenite was prepared by dissolving selenite powder in sterile PBS solution, and a final $10 \mu \mathrm{mol} / \mathrm{I}$ working solution was used in the current study (as determined previously). LY294002 was obtained from Promega (Madison, WI, USA). Manganese (III) tetrakis (1-methyl-4-pyridyl) porphyrin (MnTMPyP) was purchased from Merck Calbiochem (San Diego, CA, USA). Insulin was purchased from Roche (Auckland, NZ, USA). Actinomycin D and DAPI were purchased from Beyotime (Haimen, Jiangsu, China).

The antibody against $\beta$-actin was purchased from Sigma-Aldrich. Antibodies recognizing cleaved caspase 9, cleaved PARP, p-Src (Tyr416), Src, p-p85 (Tyr458), p85, p-PDK1 (Ser241), PDK1, p-AKT(Thr308), AKT, p-FoxO1 (Ser256), Fox01, p-FoxO4 (Ser193), FoxO4, the Pho-(Ser) 14-3-3 binding motif, PTEN, Bim, p-PTEN(Ser380), Myc-tag, VDAC and HA-tag were purchased from Cell Signaling Technology (Beverly, MA, USA). Antibodies against B23, FoxO3a and p-FoxO3a (Ser253) were obtained from Santa Cruz (Santa Cruz, CA, USA).

Immunoblotting. To prepare lysates, cells were collected after trypsinization and washed twice with cold PBS. Total cell lysates were prepared in RIPA buffer (20 mM Tris (pH 7.5), $150 \mathrm{mM} \mathrm{NaCl}, 1 \mathrm{mM}$ EDTA, 1 mM EGTA, 1\% Triton X-100, 
$2.5 \mathrm{mM}$ sodium pyrophosphate, $1 \mathrm{mM} \beta$-glycerophosphate, $1 \mathrm{mM} \mathrm{Na}_{3} \mathrm{VO}_{4}, 1 \mu \mathrm{g} / \mathrm{ml}$ leupeptin and $1 \mathrm{mM}$ PMSF). Specifically, nuclear fractions were obtained using the NE-PER nuclear/cytoplasmic extraction kit. Mitochondria were fractionated using the Mitochondria Isolation Kit for Mammalian Cells (Thermo Fisher Scientific, Rockford, IL, USA). The protein concentration in each sample was determined using the Bradford assay. After normalization, equal amounts of proteins were fractionated on $8-15 \%$ SDS-PAGE gels. The proteins were then transferred to nitrocellulose membranes (GE Healthcare, Little Chalfont, Buckinghamshire, UK) and incubated with the indicated primary antibodies and corresponding HRPconjugated secondary antibodies. The immunoreactive bands were visualized by chemiluminescence according to the manufacturer's recommendations (Thermo Fisher, Waltham, MA, USA).

Plasmids transient transfection analysis. Approximately $4 \times 10^{5}$ cells were plated into six-well plate 1 day before transfection experiments to allow for cell attachment and growth. When grown to $50 \%$ confluency, cells were transfected with indicated plasmids by lipofectamine 2000 reagent (Invitrogen), according to the protocol. Briefly, about $4 \mu \mathrm{g}$ plasmids were transfected into cells together with $5 \mu$ l lipofectamine 2000 reagent per well. At $24 \mathrm{~h}$ after transfection, cells were treated as indicated.

Small interfering RNAs. AKT1 siRNA (5'-AAGGAGGGUUGGCUGCA CAAA-3'); FOXO3a siRNA (5'-AAUGUGACA-UGGAGUCCAUUA-3'); Bim SiRNA (5'-AAGGUAGACAAUUGCAGCCUG-3'); PTEN si RNA (5'-GACUUGAAGGC GUAUACAGtt-3') and the control siRNA (5'-UUCUCCGAACGUGUCA-CGUTT3') were chemically synthesized by GenePharm (Shanghai, China). Cells cultured in six-well plates were transfected with $100 \mathrm{pM}$ siRNA by lipofectamine 2000 according to the guideline as described above. Cells were then subjected to further treatment as demanded.

Co-immunoprecipitation. Cells were harvested and total cell lysates were prepared in RIPA buffer. In all, $200 \mu \mathrm{g}$ aliquots of lysates were immunoprecipitated by the appropriate antibodies and normal immunoglobulin antibodies as a control. The immunoprecipitates were then captured by $25 \mu \mathrm{l}$ protein $\mathrm{A}+\mathrm{G}$ agarose beads (Santa Cruz). After being washed and elutioned, the immunoprecipitates were subjected to western blot assays.

PI3K kinase assay. The in vitro kinase activity of PI3K in our study was examined using the PI3-Kinase Activity Assay Kit from Echelon Biosciences (Salt Lake, UT, USA). Briefly, cells were treated as indicated, and then PI3K was immunoprecipitated from cellular lysates using PI3K antibodies. Kinase reactions were performed using PI2P as the substrate. The production of PI3P in each sample was detected using ELISA, according to the methods provided by the manufacturer. The relative activity of PI3K could be calculated based on the concentration of PI3P in each group.

AKT kinase assay. The in vitro kinase activity of AKT kinase in cells treated with or without selenite was determined using the Akt Kinase Assay Kit (Cell Signaling Technology). Briefly, cells were harvested, and cell lysates were prepared in cell lysis buffer provided by the manufacturer. Subsequently, immobilized Akt antibody was used to immunoprecipitate p-AKT from cell lysates followed by in vitro detection of Akt kinase activity using GSK-3 fusion protein and cold ATP in the kinase buffer. Finally, the activity of AKT kinase in each sample was determined according to GSK-3 $\alpha / \beta$ phosphorylation by western blotting.

In vitro phosphatase activity assay of PTEN. The phosphatase activity of PTEN was determined using the Malachite Green Assay Kit from Echelon Biosciences. Briefly, PTEN was immunoprecipitated from treated cells using PTEN monoclonal antibodies. After thorough washing and purification, the immunocomplexes were dissolved in the enzyme reaction buffer. The enzyme reaction was initiated using the PIP3 substrate provided by the manufacturer. Malachite green solution was added at the termination of the enzyme reaction. The absorbance of the solution was then read in each well. The relative conversion of PI3P to PI2P was calculated to reflect the phosphatase activity of PTEN.

RT-PCR. Total RNA was extracted using Trizol reagent (Invitrogen) according to the manufacturer's instructions. Reverse transcription was performed using M-MLV reverse transcriptase, oligo dT primer and dNTPs (Promega), and the resultant CDNA was subjected to PCR. Primers for PTEN (forward: $5^{\prime}$-CCAAT
GTTCAGTGGCGGAACT-3'; reverse: $5^{\prime}$-GAACTTGTCTTCCCGTCGTGTG-3'), Bim (forward: 5'-GAGCCACAAGACAGGAGC-3'; reverse: 5'-AAGGGCAATTCTGAGGGA-3') and GAPDH (forward: 5'-CATCTTCCAGGAGC-GAGATC-3'; reverse: $5^{\prime}$-GCTTGA-CAAAGTGGTCGTTG- $3^{\prime}$ ) were synthesized by Sangon Biotech (Shanghai Co. Ltd., China). The band intensities of each band were normalized to the corresponding GAPDH bands.

Immunofluorescence. Cells were grown on coverslips for $24 \mathrm{~h}$ before treatment with $10 \mu \mathrm{mol} / / \mathrm{l}$ selenite or PBS solution for $24 \mathrm{~h}$. Cells were then fixed in $3.7 \%$ paraformaldehyde and permeabilized with $0.1 \%$ Triton X-100 solution for $10 \mathrm{~min}$. After thorough washing with PBS three times, each for $5 \mathrm{~min}$, the samples were blocked with $2 \%$ BSA solution for 30 min at room temperature. Subsequently, slides were incubated with the indicated primary antibodies overnight at $4{ }^{\circ} \mathrm{C}$. Thereafter, cells were incubated with FITC- or Cy3-labeled secondary antibodies for $1 \mathrm{~h}$ at room temperature, followed by counterstaining with DAPI solution. Images were acquired using an Olympus laser scanning confocal FV1000 microscope (Olympus, Tokyo, Japan) and analyzed using Olympus Fluoview software. Representative images are shown in figures with identical settings.

Apoptosis assay. The percentage of cells undergoing apoptosis was determined using Annexin V/PI double staining with the Apoptosis Detection Kit from Merck Calbiochem. The assay was performed according to the manufacturer's instructions. Briefly, after performing the indicated treatments, cells were harvested and washed twice with pre-cold PBS buffer, and then cells were stained with Annexin $\mathrm{V}$ and $\mathrm{PI}$ in $1 \times$ binding buffer. The percentage of cells undergoing apoptosis was determined using an Accuri C6 flow cytometer (Accuri Cytometers Inc., Ann Arbor, MI, USA). All experiments were performed three times independently, and the results are expressed as the mean values \pm S.D.

ChIP assay. ChIP assays were performed using the SimpleChIP Enzymatic Chromatin IP Kit (Cell Signaling Technology) according to the manufacturer's instructions. Briefly, the proteins were crosslinked to DNA in live nuclei using $1 \%$ formaldehyde (Sigma-Aldrich). The crosslinked chromatin was extracted from cells using buffer A and buffer B provided in the ChIP kit and then digested using micrococcal nuclease for 20 min at $37^{\circ} \mathrm{C}$ into small fragments. The crosslinked chromatin was aliquoted and immunoprecipitated using $2 \mu \mathrm{g}$ of foxo3a antibody and normal rabbit immunoglobulin $\mathrm{G}$ antibody overnight at $4{ }^{\circ} \mathrm{C}$ with gentle rotation. The complexes were captured by ChIP-Grade Protein $\mathrm{G}$ agarose beads for $2 \mathrm{~h}$ at $4{ }^{\circ} \mathrm{C}$ with gentle rotation. Following thorough washing, bound DNA fragments were eluted and analyzed by subsequent PCR using primers for PTEN and bim as follows: PTEN: forward 5'-GCATTTCCGAATCAGCTCTCT-3'; reverse: 5'-CCAAGTGACTTATCTCTGGTCTGAG-3'; and bim: forward 5'-AGGCAGAA CAGGAGGAGA-3'; reverse 5'-AACCCGTTTGTA-AGAGGC-3'.

Immunohistochemistry evaluation. The colorectal xenograft model was established as previously described. ${ }^{8}$ All animal procedures were performed in accordance with the guidelines issued by the committee on animal research of Peking Union Medical College and approved by the institutional ethics committee. At the termination of the experiments, tumor tissues from both the control and selenitetreated groups were sectioned. Half of these samples were homogenized and subjected to western blotting as described above, whereas the remaining tissues were embedded in paraffin for immunohistochemical analysis. First, $4 \mu \mathrm{m}$ thick tissue sections were prepared on slides and then were dewaxed and rehydrated in xylene and graded alcohols. Antigen retrieval was achieved by heating the slides in a $95^{\circ} \mathrm{C}$ water bath with $0.01 \mathrm{~mol} / \mathrm{l}$ citrate buffer at $\mathrm{pH} 6.0$ for $20 \mathrm{~min}$. Subsequently, endogenous peroxidase activity was quenched by incubation in $3 \%$ hydrogen peroxide solution (Zhongshan Gold Bridge, Beijing, China). Thereafter, slides were blocked with $10 \%$ goat serum for $0.5 \mathrm{~h}$ after which the sections were incubated with primary antibodies as indicated in the figures (Figure $5 \mathrm{c}$ and Supplementary Figure S5) for $1 \mathrm{~h}$. After three sequential washes ( 5 min each) in PBS solution, these samples were incubated with a streptavidin-peroxidase complex for an additional $1 \mathrm{~h}$. All of the above procedures were performed at room temperature. Next, a diaminobenzidine working solution was applied after additional washes. Finally, the slides were counterstained with hematoxylin.

Statistical analysis. Each experiment was repeated at least three times. For all the quantitative analyses represented in the histograms, the values are expressed as the mean values \pm S.D. The significance of the differences between mean values were assessed using Student's t-test. All computations were calculated using the Microsoft Excel program. 


\section{Conflict of Interest}

The authors declare no conflict of interest.

Acknowledgements. We thank Dr Wenij Dong for his AKT plasmids and kind suggestions. This work was supported by the grants from National Natural Science Foundation for Young Scholars of China (Grant No. 31101018), National Natural Science Foundation of China (No. 31170788 and No. 30970655), State Key Laboratory Special Fund (No. 2060204) and Research Fund for the Doctoral Program of Higher Education of China (No. 20091106110025).

1. Rayman MP. Selenium and human health. Lancet 2012; 379: 1256-1268.

2. Brozmanova J. [Selenium and cancer: from prevention to treatment]. Klin Onkol 2011; 24 171-179.

3. Clark LC, Dalkin B, Krongrad A, Combs GF Jr., Turnbull BW, Slate EH et al. Decreased incidence of prostate cancer with selenium supplementation: results of a double-blind cancer prevention trial. Br J Urol 1998; 81: 730-734.

4. Clark LC, Combs GF Jr, Turnbull BW, Slate EH, Chalker DK, Chow J et al. Effects of selenium supplementation for cancer prevention in patients with carcinoma of the skin. A randomized controlled trial. Nutritional Prevention of Cancer Study Group. JAMA 1996; 276: 1957-1963.

5. Park SH, Kim JH, Chi GY, Kim GY, Chang YC, Moon SK et al. Induction of apoptosis and autophagy by sodium selenite in A549 human lung carcinoma cells through generation of reactive oxygen species. Toxicol Lett 2012; 212: 252-261.

6. Chen XJ, Duan FD, Zhang HH, Xiong Y, Wang J. Sodium selenite-induced apoptosis mediated by ROS attack in human osteosarcoma U2OS cells. Biol Trace Elem Res 2012; 145: 1-9.

7. Guan L, Han B, Li Z, Hua F, Huang F, Wei W et al. Sodium selenite induces apoptosis by ROS-mediated endoplasmic reticulum stress and mitochondrial dysfunction in human acute promyelocytic leukemia NB4 cells. Apoptosis 2009; 14: 218-225.

8. Huang F, Nie C, Yang Y, Yue W, Ren Y, Shang Y et al. Selenite induces redox-dependent Bax activation and apoptosis in colorectal cancer cells. Free Radic Biol Med 2009; 46: 1186-1196.

9. Luo H, Yang Y, Huang F, Li F, Jiang Q, Shi K et al. Selenite induces apoptosis in colorectal cancer cells via AKT-mediated inhibition of beta-catenin survival axis. Cancer Lett 2012; 315: 78-85.

10. Lam EW, Francis RE, Petkovic M. FOXO transcription factors: key regulators of cell fate. Biochem Soc Trans 2006; 34 (Pt 5): 722-726.

11. van der Vos KE, Gomez-Puerto C, Coffer PJ. Regulation of autophagy by Forkhead box (FOX) O transcription factors. Advances Biol Regul 2012; 52: 122-136.

12. Fu Z, FOXOs TindallDJ. cancer and regulation of apoptosis. Oncogene 2008; 27 2312-2319.

13. Zhang Y, Gan B, Liu D, Paik J-h. FoxO family members in cancer. Cancer Biol Ther 2011; 12: 253-259.

14. Arden $\mathrm{KC}$. Multiple roles of FOXO transcription factors in mammalian cells point to multiple roles in cancer. Exp Gerontol 2006; 41: 709-717.

15. Calnan DR, Brunet A. The FoxO code. Oncogene 2008; 27: 2276-2288.

16. Tran H, Brunet A, Griffith EC, Greenberg ME. The many forks in FOXO's road. Sci STKE 2003; 2003: RE5.

17. Dudgeon $\mathrm{C}$, Wang $\mathrm{P}$, Sun $\mathrm{X}$, Peng $\mathrm{R}$, Sun $\mathrm{Q}, \mathrm{Yu} \mathrm{J}$ et al. PUMA induction by FoxO3a mediates the anticancer activities of the broad-range kinase inhibitor UCN-01. Mol Cancer Ther 2010; 9: 2893-2902.

18. Stahl M, Dijkers PF, Kops GJ, Lens SM, Coffer PJ, Burgering BM et al. The forkhead transcription factor FoxO regulates transcription of p27Kip1 and Bim in response to IL-2. J Immunol 2002; 168: 5024-5031.

19. Roy HK, Olusola BF, Clemens DL, Karolski WJ, Ratashak A, Lynch HT et al. AKT protooncogene overexpression is an early event during sporadic colon carcinogenesis. Carcinogenesis 2002; 23: 201-205.

20. Altomare DA, Testa JR. Perturbations of the AKT signaling pathway in human cancer Oncogene 2005; 24: 7455-7464.

21. Tsai KL, Sun YJ, Huang CY, Yang JY, Hung MC, Hsiao CD. Crystal structure of the human FOXO3a-DBD/DNA complex suggests the effects of post-translational modification. Nucleic Acids Res 2007; 35: 6984-6994.

22. Cao TM, Hua FY, Xu CM, Han BS, Dong H, Zuo L et al. Distinct effects of different concentrations of sodium selenite on apoptosis, cell cycle, and gene expression profile in acute promyeloytic leukemia-derived NB4 cells. Ann Hematol 2006; 85: 434-442.

23. Chiacchiera F, Matrone A, Ferrari E, Ingravallo G, Lo Sasso G, Murzilli $S$ et al. p38 blockade inhibits colorectal cancer growth in vivo by inducing a switch from $\mathrm{HIF} 1 \alpha$ - to FoxO-dependent transcription. Cell Death Differ 2009; 16: 1203-1214.

24. Li Y, Prasad A, Jia Y, Roy SG, Loison F, Mondal S et al. Pretreatment with phosphatase and tensin homolog deleted on chromosome 10 (PTEN) inhibitor SF1670 augments the

efficacy of granulocyte transfusion in a clinically relevant mouse model. Blood 2011; 117: $6702-6713$.

25. Brozmanova J, Manikova D, Vlckova V, Chovanec M. Selenium: a double-edged sword for defense and offence in cancer. Arch Toxicol 2010; 84: 919-938.

26. Xiang N, Zhao R, Zhong W. Sodium selenite induces apoptosis by generation of superoxide via the mitochondrial-dependent pathway in human prostate cancer cells. Cancer Chemother Pharmacol 2009; 63: 351-362.

27. Kralova V, Benesova S, Cervinka M, Rudolf E. Selenite-induced apoptosis and autophagy in colon cancer cells. Toxicol In Vitro 2012; 26: 258-268.

28. Carter ME, Brunet A. FOXO transcription factors. Curr Biol 2007; 17: R113-R114.

29. Sykes Stephen M, Lane Steven W, Bullinger L, Kalaitzidis D, Yusuf R, Saez B et al. AKT/ FOXO signaling enforces reversible differentiation blockade in myeloid leukemias. Cell 2011; 146: 697-708.

30. Yang J-Y, Zong CS, Xia W, Yamaguchi H, Ding Q, Xie X et al. ERK promotes tumorigenesis by inhibiting FOXO3a via MDM2-mediated degradation. Nat Cell Biol 2008; 10: $138-148$

31. Hu MC, Lee DF, Xia W, Golfman LS, Ou-Yang F, Yang JY et al. IkappaB kinase promotes tumorigenesis through inhibition of forkhead FOXO3a. Cell 2004; 117: 225-237.

32. Wang X, Chen WR, Xing D. A pathway from JNK through decreased ERK and Akt activities for FOXO3a nuclear translocation in response to UV irradiation. J Cell Physiol 2012; 227: $1168-1178$

33. Brunet A, Bonni A, Zigmond MJ, Lin MZ, Juo P, Hu LS et al. Akt promotes cell survival by phosphorylating and inhibiting a Forkhead transcription factor. Cell 1999; 96: 857-868.

34. Sharma G, Kar S, Palit S, Das PK. 18ß-glycyrrhetinic acid induces apoptosis through modulation of Akt/FOXO3a/Bim pathway in human breast cancer MCF-7 cells. J Cell Physiol 2012; 227: 1923-1931.

35. Li Y, Wang Z, Kong D, Li R, Sarkar SH, Sarkar FH. Regulation of Akt/FOXO3a/GSK-3/AR signaling network by isoflavone in prostate cancer cells. J Biol Chem 2008; 283 : 27707-27716

36. Sunters A, Madureira PA, Pomeranz KM, Aubert M, Brosens JJ, Cook SJ et al. Paclitaxelinduced nuclear translocation of $\mathrm{FOXO} 3 \mathrm{a}$ in breast cancer cells is mediated by $\mathrm{c}$-Jun NH2-terminal kinase and Akt. Cancer Res 2006; 66: 212-220.

37. O'Connor L, Strasser A, O'Reilly LA, Hausmann G, Adams JM, Cory S et al. Bim: a novel member of the Bcl-2 family that promotes apoptosis. EMBO J 1998; 17: 384-395.

38. Strasser A, Puthalakath $\mathrm{H}$, Bouillet P, Huang DC, O'Connor L, O'Reilly LA et al. The role of bim, a proapoptotic BH3-only member of the Bcl-2 family in cell-death control. Ann NY Acad Sci 2000; 917: 541-548.

39. Dijkers PF, Medema RH, Lammers JW, Koenderman L, Coffer PJ. Expression of the proapoptotic Bcl-2 family member Bim is regulated by the forkhead transcription factor FKHRL1. Curr Biol 2000; 10: 1201-1204.

40. Hollander MC, Blumenthal GM, Dennis PA. PTEN loss in the continuum of common cancers, rare syndromes and mouse models. Nat Rev Cancer 2011; 11: 289-301.

41. Salmena L, Carracedo A, Pandolfi PP. Tenets of PTEN tumor suppression. Cell 2008; 133: 403-414

42. Berggren M, Sittadjody S, Song Z, Samira JL, Burd R, Meuillet EJ. Sodium selenite increases the activity of the tumor suppressor protein, PTEN, in DU-145 prostate cancer cells. Nutr Cancer 2009; 61: 322-331.

43. Ma J, Sawai H, Ochi N, Matsuo Y, Xu D, Yasuda A et al. PTEN regulates angiogenesis through PI3K/Akt/VEGF signaling pathway in human pancreatic cancer cells. Mol Cell Biochem 2009; 331: 161-171.

44. Li D, Qu Y, Mao M, Zhang X, Li J, Ferriero D et al. Involvement of the PTEN-AKT-FOXO3a pathway in neuronal apoptosis in developing rat brain after hypoxia-ischemia. $J$ Cereb Blood Flow Metab 2009; 29: 1903-1913.

45. Ferber EC, Peck B, Delpuech O, Bell GP, East P, Schulze A. FOXO3a regulates reactive oxygen metabolism by inhibiting mitochondrial gene expression. Cell Death Differ2011; 19: 968-979

46. Yoon SO, Park SJ, Chung AS. Selenite inhibits apoptosis via activation of the PI3-K/Akt pathway. Ann NY Acad Sci 2002; 973: 221-223.

47. Zhu X, Guo K, Lu Y. Selenium effectively inhibits 1,2-dihydroxynaphthalene-induced apoptosis in human lens epithelial cells through activation of PI3-K/Akt pathway. Mol Vis 2011; 17: 2019-2027.

48. Guan L, Han B, Li J, Li Z, Huang F, Yang Y et al. Exposure of human leukemia NB4 cells to increasing concentrations of selenite switches the signaling from pro-survival to proapoptosis. Ann Hematol 2009; 88: 733-742.

(c)

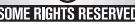
Share Alike 3.0 Unported License. To view a copy of this license, visit http://creativecommons.org/licenses/by-nc-sa/3.0/ 